\title{
IMPROVING B-CELL DEPLETION IN RHEUMATOID ARTHRITIS AND SYSTEMIC LUPUS ERYTHEMATOSUS
}

Authors: Pedro Mota' ${ }^{1}$, Venkat Reddy ${ }^{2}$,David Isenberg ${ }^{3}$

1. Department of Internal Medicine, Hospital da Luz, Lisbon, Portugal (pedro.mota@hospitaldaluz.pt)

2. Centre for Rheumatology, Division of Medicine, University College London (v.reddy@ucl.ac.uk)

3. Centre for Rheumatology, Division of Medicine, University College London (d.isenberg@ucl.ac.uk)

Corresponding author:

Professor David Isenberg

Room 424

The Rayne Institute

5 University Street

London

WC1E 6JF

Tel: $\quad 02031082148$

Fax: $\quad 02031082152$

Email: Centre for Rheumatology, Division of Medicine, University College Hospital d.isenberg@ucl.ac.uk 


\section{SUMMARY}

Introduction: Rituximab-based B-cell depletion (BCD) therapy is effective in refractory RA and, although used to treat patients with refractory SLE in routine clinical practice, rituximab failed to meet the primary endpoints in two large randomised controlled trials of non-renal (EXPLORER) and renal (LUNAR) SLE.

Areas covered: We review how BCD could be improved to achieve better clinical responses in RA and SLE. Insights into the variability in clinical response to BCD in RA and SLE, may help develop new therapeutic strategies. To this end, a literature search was performed using the following terms: rheumatoid arthritis, systemic erythematosus lupus, rituximab and B-cell depletion..

Expert Commentary: Poor trial design may have, at least partly, contributed to the apparent lack of response to BCD in the two RCTs of patients with SLE. Enhanced B-cell depletion and/or sequential therapy with Belimumab may improve clinical response at least in some patients with SLE 


\section{IMPROVING B-CELL DEPLETION IN RHEUMATOID ARTHRITIS AND SYSTEMIC LUPUS ERYTHEMATOSUS}

\section{Introduction}

Rheumatoid Arthritis (RA) and Systemic Lupus Erythematosus (SLE) are two systemic autoimmune diseases that arise from a complex interplay of genetic and environmental factors.

The fundamental role of B-cells in the pathogenesis of RA and SLE has been reviewed, previously, and autoantibodies are characteristically associated with both RA and SLE 1,2. It has been suggested that interaction between B cells and T cells perpetuates a "vicious cycle" of inflammation ${ }^{1,2}$. B cells can act as antigen presenting cells providing signals to T cells, which in turn provide "help" to other B cells through the delivery of cytokines and cell-surface ligands ${ }^{3-5}$. Based on this, it could be hypothesised that elimination of either B cells or $T$ cells should break the vicious cycle of inflammation, thereby, restoring a disease-free state, at least temporarily. Early studies in the 1990s, centred on the consideration that $\mathrm{T}$ cells played the dominant role, targeted T-cells. However, the results were disappointing in both RA and SLE 6,7. Alternatively, B-cell targeting as a therapeutic option for RA was first proposed in the mid 1990s founded on the hypothesis that autoantibodies, such as Rheumatoid Factor may in turn promote the survival of B cells, thus, propagating chronic inflammation in RA 8 .

2. Why were B-cells targeted in Rheumatoid Arthritis and Systemic Lupus Erythematosus? 
Targeting of $B$ lymphocytes is of particular interest since B-cells play a major role in the pathogenesis of RA and SLE. B-cells develop from pluripotent stem cells in the bone marrow each expressing B-cell receptors with unique immunoglobulin variable region. Their maturation and activation processes occur as they migrate through the blood to perifollicular, germinal-centre and memory compartments in the lymph nodes and spleen and finally, as plasma cells, to the bone marrow. Delivery of survival and trophic signals such as cellsurface ligands (such as vascular cell-adhesion molecule 1), or soluble factors, such as B-cell activating factor (BAFF, also known as BLyS) appear to be important for B-cell survival and maturation ${ }^{9}$. Autoantibody production by pathogenic B- lineage cells may be sustained by long-lived plasma cells ${ }^{10}$. In addition to antibody-dependent mechanisms, B cells also act as antigenpresenting cells and co-stimulate T Cells and other inflammatory cell types ${ }^{5}$.

In RA, leukocytes infiltrate the synovial compartment contributing to synovitis. Even though $T$ cells are abundant in the synovial milieu, direct targeting of $T$ cells by cyclosporine or other $T$ cell depleting agents has shown limited or no efficacy ${ }^{11}$. The synovium of RA patients contains abundant myeloid cells and plasmacytoid dendritic cells, whose cytokines, HLA class II and co-stimulatory molecules are essential for T-cell activation and antigen presentation. Synovial $B$ cells are mainly part of T-cell-B-cell aggregates which are supported by expression of factors such as 'A proliferation-inducing ligand' (APRIL), BAFF, and chemokines ${ }^{11}$. As key factors of humoral adaptive immunity, these molecules constitute potential treatment targets for RA, discussed later.

In SLE, several studies have focused on the role of anti-dsDNA antibodies in lupus nephritis. An interesting proposition is that the binding of antibodies to dsDNA itself is not the most critical determinant of tissue damage. Instead, extracellular double-stranded DNA appears mainly in the form of nucleosomes, as fragments of chromatin released by apoptotic cells and that anti-dsDNA antibodies bind these circulating nucleosome forming antibody-nucleosome complexes, which in turn bind the renal glomerular basement membrane triggering complement-mediated glomerulonephritis. This process may be the result of a "charge interaction", where once anti-nucleosome complexes have formed in the circulation, the histones (part of nucleosome), being positively 
charged, bind to the negatively charged components of the glomerular basement membrane (GBM). Thus, histones in effect act as a bridge between the anti-DNA/nucleosome antibodies and the GBM. This is known as the "histone-bridge theory" ${ }^{12}$. Therefore, anti-dsDNA antibodies may mediate tissue damage by direct antibody- and complement-mediated cytotoxicity.

These observations indicating a key role of $B$ cells in propagating inflammation in RA and SLE, provide the rationale for targeting $B$ cells in patients with RA and SLE ${ }^{1,3}$.

\section{B-cell depletion agents}

\subsection{Rituximab as B-cell Depletion Agent in Autoimmune Disease}

Rituximab (RTX) is a chimeric monoclonal antibody that selectively binds the target antigen, CD20, and induces B-cell depletion. The safety and efficacy of its use in $B$ cell malignancies resulted in licensing for the treatment of nonHodgkin Lymphoma ${ }^{13}$. CD20 is expressed on B lymphocytes from the pre-B stage to the mature $B$ stage, but not plasma cells ${ }^{1}$, and therefore, treatment with rituximab would directly target only CD20+ B-cells.

Edwards and Cambridge pioneered B-cell depletion therapy in 1999 for the treatment of $\mathrm{RA}^{14}$. Following the successful pivotal phase III study ${ }^{15}$, rituximab was approved by National Institute for Health and Care Excellence (NICE) in 2007. Isenberg and colleagues led studies on B-cell depletion therapy with rituximab for the treatment of Lupus ${ }^{16}$. In 2004, the first phase $1 /$ II dose escalation trial of rituximab in SLE patients with active disease reported a favourable safety profile and promising clinical efficacy ${ }^{17}$. Although widely used in routine practice for the treatment of refractory SLE, with many case reports published indicating its utility ${ }^{18,19}$, rituximab did not meet endpoints in two randomized controlled trials: LUNAR and EXPLORER ${ }^{20,21}$. However, these 
trials have been criticised for their poor design 22 23, 24 . See later for more detailed discussion.

\subsection{Rituximab - Risk and Benefit Consideration:}

The use of rituximab in combination with immunosuppressants has raised concerns about increased risk of serious infection. However, most of the side effects reported with the use of rituximab were mild respiratory and urinary infections ${ }^{25}$. A significant reduction in autoantibody levels after B-cell depletion, namely rheumatoid factor (IgM, IgG and $\lg A$ isotypes) and antibodies to citrullinated peptides occur in contrast to a small reduction in total immunoglobulins levels and antibodies to pneumococcal-polysaccharide ${ }^{10}$. Albert et al. have reported that response to immunisation following BCD with rituximab may be impaired in patients with SLE ${ }^{26}$. Therefore, vaccination against influenza and pneumococcus prior to B-cell depletion is recommended 27. In our cohort of patients with SLE, we have recently described that low serum immunogloblulin levels after rituximab therapy seems to be largely restricted to the IgM isotype particularly in those receiving concomitant and/or sequential therapy with mycophenolate mofetil ${ }^{28}$. Although rare, late-onset neutropenia has been reported with Rituximab ${ }^{27}$. Therefore, careful monitoring of patients with optimal dosage of immunosuppressants such as mycophenolate mofetil is essential to minimise the risk of infections after BCD with rituximab.

Infusion-related reactions, mostly mild and manageable, associated with rituximab were reported in $20-40 \%$ of patients and premedication with antihistamine and methylprednisolone may help reduce the risk of infusionrelated reactions ${ }^{16,27}$.

Progressive multifocal leukoencephalopathy (PML) has been reported after treatment with rituximab in patients with RA who had other comorbidities ${ }^{29}$ and, also in patients with SLE treated with conventional immunosuppressants alone 27. Thus, the absolute risk of PML associated with rituximab appears to be low. 


\subsection{Rituximab in Rheumatoid Arthritis:}

In 1999, the first pilot study of rituximab-based B-cell depletion therapy was initiated at University College London Hospital to treat patients with refractory RA ${ }^{14}$. The length of benefit from B-cell depletion was related to the dose of rituximab, with shorter responses at low rituximab dose. These early studies demonstrated preliminary safety and efficacy of rituximab in RA ${ }^{30}$ and informed the design of the pivotal phase III randomized, double-blind, controlled study for patients with RA ${ }^{15}$. The regimen used in this study was two doses $(1000 \mathrm{mg})$ of rituximab given 2 weeks apart, with premedication including a single $100 \mathrm{mg} \mathrm{IV}$ methylprednisolone and oral prednisolone (60 $\mathrm{mg}$ on day 2 and days 4 to 7,30 mg per day on day 8 to 14). Patients considered to be responders were retreated at or just before predicted relapse. This study showed that rituximab was effective in methotrexate-refractory RA ${ }^{15}$. Almost $80 \%$ of subjects showed benefit and a significant proportion of patients achieved major benefit as assessed by ACR50 criteria $^{15}$.

A significant improvement was noted in extra-articular features such as subcutaneous nodules, vasculitis, anaemia and amyloidosis ${ }^{30,31}$. Edwards et at. described a mean time to retreatment of 20 months, with most of patients previously refractory to standard disease-modifying antirheumatic drugs being satisfactorily controlled by methotrexate, sulphasalazine, or leflunomide for at least 1 year ${ }^{31}$. Clinical response in patients with RA lasted the period of B-cell depletion, which typically lasts $7-8$ months ${ }^{1}$. B-Cell repopulation occurs with a immature phenotype, as seen after bone marrow transplantation or in umbilical cord blood ${ }^{32}$.

Repeated treatment with rituximab in RA resulted in reduction of serum Creactive protein (CRP) levels from $50-100 \mathrm{mg} / \mathrm{L}$ to less than $2 \mathrm{mg} / \mathrm{L}$ (reference range $<5 \mathrm{mg} / \mathrm{L}$ ) and, clinical improvement and reduction in CRP were closely correlated with decrease in antibody levels ${ }^{1}$. Furthermore, almost $50 \%$ of patients achieved clinical benefit that persisted even after repopulation of $B$ cells, with the longest recorded period of improvement being 42 months $^{31}$. Serum RF levels fell over a period of months, with IgG RF decreasing by $60 \%$ and antibodies to citrullinated peptides also decreased, becoming undetectable 
in some cases ${ }^{10}$. In the UCL RA patient cohort, clinical relapse has invariably been preceded by a detectable rise in autoantibodies ${ }^{10}$. A potential resistance mechanism of rituximab therapy though is that the underlying immunopathological process might be reactivated through signals from autoantibodies produced by long-lived plasma cells, which are not targeted by rituximab ${ }^{31}$.

\subsection{Rituximab in SLE:}

Leandro et al reported the first open uncontrolled study of rituximab for SLE patients in $2002{ }^{16}$. Since then, several groups have reported on the efficacy of rituximab treatment in SLE patients with an impressive clinical response in 171 (91\%) of 188 adults with active, multi-organ SLE refractory to corticosteroid and immunosuppressive therapy ${ }^{33}$. Looney and colleagues reported in their phase II study about the safety and efficacy of rituximab ${ }^{17}$. In the uncontrolled studies, two rituximab regimens were used: two $1000 \mathrm{mg}$ doses 2 weeks apart and 375 $\mathrm{mg} / \mathrm{m}^{2}$ for 4 weeks with the latter regimen showing a higher rate of response with 4 weeks regimen (94 vs $83 \%, p=0.048$ ). In a systematic review of 300 patients with SLE from 21 studies with a follow-up period ranged from 3 months to over 36 months, Gregersen and Jayne observed that eGFR $<60$ $\mathrm{mL} / \mathrm{min} / 1.73 \mathrm{~m}^{2}$ and nephrotic syndrome were associated with poor prognosis ${ }^{34}$. Therefore In patients with nephrotic syndrome, rituximab may be excreted more rapidly and more frequent doses of rituximab may be required.

The response to $\mathrm{B}$-cell depletion is remarkably variable between patients with SLE, with respect to the degree and duration of $B C D$, clinical response, duration of remission and relapse ${ }^{35}$. Treatment with rituximab is associated with a reduction in anti-dsDNA and anti-nucleosome antibody levels, but no change is seen in anti-ENA antibodies ${ }^{36}$. These findings suggest that antidsDNA and anti-nucleosome antibodies, at least in a proportion of patients, are secreted by short-lived plasma cells, whereas anti-ENA antibodies are secreted by long-lived plasma cells ${ }^{10}$. 
Vital et al. described that effectiveness of B-cell depletion after Rituximab infusion in 39 SLE patients, using highly sensitive flow cytometry ${ }^{37}$. They reported a significant reduction from baseline BILAG, major clinical response and partial clinical response rates of $51 \%$ and $31 \%$, respectively. Fifty percent of the patients relapsed after 6-18 months. B cells were detectable in peripheral circulation in 21 patients after 2 infusions of rituximab, which included all 7 nonresponders. Memory B cell and plasmablast frequency in peripheral blood at 26 weeks after rituximab was significantly higher in patients with earlier relapse versus patients with later relapse ${ }^{37}$.

Unexpectedly, the two randomized clinical trials (LUNAR [19] and EXPLORER [10]) investigating the safety and efficacy of rituximab failed to meet their primary endpoints. The apparent lack of response to rituximab in these two trials was attributed to poor design ${ }^{23}$, particularly concomitant therapy with high doses of corticosteroids, which may have interfered with the detection of clinical response attributable to rituximab specifically ${ }^{33}$. However, LUNAR trial post-hoc analysis also showed a trend toward clinically relevant benefit in Africa-American and Hispanic patients with lupus nephritis with none of the patients treated with rituximab requiring rescue therapy with cyclophosphamide 38. Further, post-hoc analyses revealed improvements in clinical and laboratory parameters, including anti-dsDNA antibody and serum C3 levels ${ }^{24}$. Moreover, B-cell depletion clinical trials on Lupus Nephritis patients may need a longer follow-up given the data from a long-term follow-up study showing a significant number of patients achieve complete response during the second year of follow-up ${ }^{39}$.

Conventional standard of care for patients with SLE who have renal involvement includes corticosteroids, the prolonged use of which at high doses is associated with irreversible adverse effects such as diabetes, hypertension, weight gain and osteoporosis. Therefore, Lightstone et al proposed a rituximab based steroid sparing regimen to treat patients with lupus nephritis demonstrating remarkable efficacy ${ }^{40}$. Isenberg and co-workers at UCLH, employed similar treatment regimen to treat SLE patients with a short duration of diagnosis whose principal clinical features were non-renal, again revealing steroid-sparing effect of rituximab ${ }^{41}$. The results of this study showed that B-cell 
depletion is effective when used early and can reduce long-term steroid burden (6 months cumulative mean post-B-cell depletion $1287.3 \mathrm{mg}$ vs $2834.6 \mathrm{mg}$ of matched controls). Recently, Lightstone and her colleagues have reported data from the first 50 consecutive patients with biopsy-proven active International Society of Nephrology/Renal Pathology Society (ISN/RPS) class III, IV, or class $\checkmark$ LN, treated with RITUXILUP protocol without long-term oral steroids. They reported that B-cell depletion at the time of diagnosis enabled 48 out of 50 patients to avoid the use of maintenance oral steroids at 2 years of follow-up ${ }^{42}$. Disappointingly, owing to recruitment difficulties, a randomised non-inferiority open multicentre trial, entitled RITUXILUP (NCT01773616) established to investigate the validity of rituximab-based steroid-sparing protocol for treating patients with SLE with refractory lupus nephritis has been discontinued.

Therefore, a better understanding of the underlying reasons for the observed discrepancy in the efficacy of rituximab between individuals with SLE and, also between uncontrolled and controlled studies, may provide insights to develop strategies to target $B$ cells more effectively.

\subsection{Variability in Biologic Response to Rituximab}

\subsubsection{Rituximab Resistance vs Refractory Disease}

It is recognized that even effective depletion as measured in peripheral blood does not always correlate with clinical response ${ }^{37}$. As previously suggested by our group, to understand these discrepancies better it is important to distinguish inadequate clinical response despite effective depletion as "refractory disease", and not achieving effective depletion as "rituximab resistance" ${ }^{35}$. The latter could be explained by insufficient dose or increased drug clearance, but also to resistance of B-cell clones to depletion mechanisms induced by rituximab. However, failure to achieve clinical response despite effective depletion may occur because the disease is not B-cell dependent for perpetuation. These important concepts led us to hypothesize that patients who do not achieve clinical response but are effectively depleted ("refractory disease") and who do not reveal any evidence of early repopulation may not benefit from further B-cell targeted therapies. In contrast, at least some patients from the group with poor 
clinical response who did not achieve effective sustained depletion ("rituximab resistance") may benefit from other B-cell depletion agents or treatment regimens ${ }^{35}$.

Another important factor to consider is the potential consequences of $B C D$ on IL-10 secreting B cells or Bregs. Our laboratory has shown that the frequency and/or function of Bregs, as assessed in in vitro studies, appears to be important in both RA ${ }^{43}$ and SLE ${ }^{44}$. However, to our knowledge as discussed in previous sections, better clinical response to rituximab is observed in patients with RA and SLE who achieve complete and durable B-cell depletion and poor response is associated with incomplete B-cell depletion ${ }^{37,45}$. In RA, clinical response to rituximab appears to relate to the degree of B-cell depletion ${ }^{46}$ and that poor clinical response to rituximab may be improved by delivering an extra dose of rituximab ${ }^{47}$. Nonetheless, further in vivo studies would provide our understanding of the clinical relevance of Bregs in the context of clinical response to $B C D$.

\subsubsection{Response to treatment}

In the initial UCL cohort of RA patients the time to relapse from repopulation of $\mathrm{B}$ cells varied from 0 to 17 months ${ }^{32}$. Repopulation of the peripheral blood occurs predominantly with naive mature and transitional $B$ cells, similar to the situation after bone marrow transplantation. As previously reviewed, prolonged clinical response in RA and in SLE seems to be associated with delayed reconstitution of peripheral blood $\mathrm{CD} 27^{+}$memory $\mathrm{B}$ cells, whereas shorter responses correlates with repopulation with higher levels of memory B cells ${ }^{35}$. Changes in serum autoantibodies also seem to correlate with clinical response. It is recognized for example that seropositive RA patients respond better than seronegative RA patients. In SLE prolonged response was also associated with baseline seronegativity for autoantibodies against extractable nuclear antigens (36), whereas patients with high dsDNA tended to flare earlier. Recently, we have described a clear disparity in fluctuations of the levels of serum $\lg G$ and anti-dsDNA autoantibodies in our cohort ${ }^{28}$. Taken together, these findings suggest that incomplete B-cell depletion and repopulation predominantly with 
memory B-cells and plasma cells is clinically relevant. In this context, it has been suggested that repopulation with IL-10 secreting B cells (regulatory B cells) may help achieve durable clinical response after rituximab, perhaps by facilitating a better balance between effector and regulatory B-cell subpopulations at repopulation after rituximab.

Clinical response to rituximab does not seem to differ between RA patients of different ethnicities. In contrast, a subgroup analysis in the LUNAR study of patients with lupus nephritis, Hispanic and African-American patients showed a more favourable response to rituximab than Caucasians.

The development of human antichimeric antibody (HACA) are described in both RA and SLE 15, 17, 48. Interestingly, patients with autoimmune diseases are more likely to develop HACAs than patients with lymphoma $(<1 \%$ in B-cell malignancies, $5 \%$ in RA ${ }^{15}$ and $26 \%$ in SLE ${ }^{20}$. However, it does not seem to influence the clinical response in RA and SLE significantly.

The potential of rituximab pharmacokinetics as a potential cause for the variability in B-cell depletion in RA and SLE has also been investigated. Serum rituximab levels, despite the same dosing regimen employed, were significantly lower in patients with SLE compared to RA. In RA, there is no evidence for correlation between serum rituximab levels and clinical response. At both 1 and 3 months after rituximab therapy, serum rituximab levels were highly variable in patients with RA and SLE ${ }^{49}$. In addition, serum rituximab levels achieved in RA patients were $>9$ fold greater compared to patients with SLE, at both 1 and 3 months after treatment with rituximab. Patients with RA, but not SLE, achieving complete depletion had significantly higher serum rituximab levels than those with incomplete B-cell depletion. The lack of correlation between rituximab levels and CD19+ B cell counts in peripheral circulation raise the possibility of intrinsic resistance of some $B$ cells to cytotoxic effects of rituximab ${ }^{49}$. Thus, the underlying reasons for the inter-individual variability in rituximab levels within each disease category remain elusive. 


\section{How can we improve B-cell depletion}

\subsection{Reflections on defining B-cell depletion}

B-cell depletion as measured by arbitrarily defined peripheral CD19+ cells $<5-10$ cells $/ \mu$ in most studies does not consistently correlate with clinical response. In contrast, the use of highly sensitive flow cytometry (HSFC) to count B-cells in both RA and SLE show that the degree of B-cell depletion correlates with clinical response, suggesting the utility of HSFC in predicting outcome ${ }^{37}$. Another important finding of studies with HSFC was that peripheral B-cell depletion was incomplete in $18 \%$ of patients with RA and $54 \%$ of patients with SLE, comprising of plasmablasts/plasma cells and memory B cells. Taken together, these findings suggest that incomplete depletion and repopulation predominantly with memory B cells and plasma cells is detectable by HSFC.

\subsection{Anti-CD20 mAbs: how do they differ and what's the relevance in RA} and SLE?

Historically, rituximab has set the bench-mark for anti-CD20mAbs and to date, rituximab remains the most successful anti-CD20 mAb that has been used in clinical practice, in both B cell malignancies and autoimmune disease. However, as discussed earlier, the variability in clinical response to rituximab in both RA and SLE highlights the patient's unmet need. To this end, research has focused on increasing the efficiency of anti-CD20 mAbs in an effort to improve the clinical response, particularly in the significant minority of patients that respond less well to rituximab. Although research in this field was driven by the need for improved B-cell depleting therapies in B cell malignancies 50, given the increasing use of B-cell depletion therapy in a range of autoimmune disease it is clinically relevant to understand whether, if any, B-cell depletion agents, based on their 'mechanistic selling points', offer specific advantages for 
considering their use in autoimmune disease such as RA and SLE, reviewed recently ${ }^{51}$.

Anti-CD20 mAbs evoke B-cell cytotoxicity through antibody-dependent cell cytotoxicity (ADCC), antibody-dependent cell phagocytosis (ADCP), complement-mediated cellular cytotoxicity (CDC) and induction of direct cell death ${ }^{50}$, illustrated in Figure 1. In order to overcome the potentially significant problem of human anti-chimeric antibodies (HACAs) associated with the use of chimeric anti-CD20 mAbs such as rituximab, humanized anti-CD20 mAbs such as Ocrelizumab and Ofatumumab were tested in clinical trial settings in RA and SLE (Ocrelizumab only).

\subsubsection{Consideration of fully humanized anti-CD20}

Ocrelizumab is a humanized anti-CD20 monoclonal antibody studied in clinical trials for RA and SLE. In RA, ocrelizumab was effective in reducing signs, symptoms and joint damage when added to methotrexate (two regimens used: $200 \mathrm{mg}$ and $500 \mathrm{mg} \times 2$ every 6 months). However, its use was associated in RA patients with a dose dependent increased risk of serious infections. Two clinical trials were initiated to test its safety and efficacy in SLE. These studies used a higher dose of ocrelizumab compared to RA trials (either 400 or 1000 mg $x 2$ at entry with repeat, single dosing every 4 months). The BEGIN study for nonrenal SLE patients was discontinued early. The BELONG study for proliferative lupus nephritis compared $1000 \mathrm{mg}$ or $400 \mathrm{mg}$ ocrelizumab at 1 day and 15 days, then repeated with a single dose every 4 months (on a maintenance therapy of high-dose glucocorticoids and either mycophenolate mofetil or cyclophosphamide dosed according to EUROLUPUS protocol). This trial was also terminated early due to a greater risk of serious infection in the ocrelizumab patients mainly in the Far East ${ }^{23}$.

Ofatumumab, another humanized anti-CD20 mAb that binds a unique epitope on the smaller of the two extracellular loops of CD20 distinct from that of rituximab, has remarkably slow off-rate (i.e dissociative half-life) and evokes potent CDC. These two features may explain its capacity to lyse rituximab- 
resistant chronic lymphocytic leukaemia targets ${ }^{52}$. Furthermore, it was shown to be effective in patients with RA who failed conventional disease modifying therapy ${ }^{53}$ and also in a patient with SLE, who had a prior history of serumsickness like reaction to rituximab ${ }^{54}$.

Thus, although there are no directly comparative data on their efficacy in both RA and SLE relative to that achieved by rituximab, the available data thus far, does not indicate that simply switching to humanized anti-CD20 mAbs improves efficacy of B-cell depletion therapy with these agents in RA and SLE. However, humanized anti-CD20 mAbs such as ocrelizumab and ofatumumab are useful in individual patients with a history of serum sickness like reaction ${ }^{54}$ and/or evidence of high titre HACAs associated with rituximab.

Other developments in anti-CD20 mAbs have focused on improving the efficiency of anti-CD20 mAbs by increasing the affinity for target antigen CD20 and/or FcyRs on effector cells. For example, AME-330, has been engineered with improved affinity for both CD20 and also FcyRIII ${ }^{55}$. The results of clinical trials of AME-330 in SLE are awaited.

Mechanistically, there are two types of anti-CD20 mAbs. Type I anti-CD20 mAbs like rituximab, are efficient at recruiting complement for cellular cytotoxicity, owing to their ability to cluster CD20:anti-CD20 mAb complexes on B cell surface. However, an undesirable consequence of clustering CD20:antiCD20 mAb complexes is their interaction with the inhibitory Fc $\gamma \mathrm{RIlb}$ on B cells, which increases the potential for internalization of these complexes leaving less amount of anti-CD20 mAb remaining bound to the surface of $B$ cells for engaging with FcyRIII-bearing effector cells ${ }^{56,57}$. Type II anti-CD20 mAbs such as Obinutuzumab does not appear to cluster CD20 and are therefore less rapidly internalized compared to rituximab, as shown in malignant and healthy $B$ cells, in vitro ${ }^{57}$. Obinutuzumab was also generated to bear an afucosylated Fc portion that engages Fc $\gamma$ RIIla with a greater affinity than that of glycosylated anti-CD20 mAbs. Thus, the type II nature and afucosylated Fc portion may explain why obinutuzumab is less efficient at evoking CDC, but more efficient at recruiting Fc $\gamma$ R-bearing effector cells to induce ADCC and ADCP. Based on, the safety and superior clinical responses in patients with chronic lymphocytic 
leukaemia (a B-cell malignancy) treated with obinutuzumab compared to those treated with rituximab ${ }^{58}$, and the observations that, in vitro, $B$ cells from patients with RA and SLE internalize rituximab impairing its efficiency of B-cell depletion ${ }^{59}$, obinutuzumab has recently entered clinical trials for the treatment of refractory SLE to explore whether the mechanistic advantages afforded by obinutuzumab will translate into clinical benefit in patients with refractory SLE.

It is not entirely clear as to what effector mechanisms are important for attaining good clinical response to BCD with rituximab. The association between higher affinity FcRIlla genotype and better clinical response to rituximab suggests that $A D C C$ appears to be a clinically relevant effector mechanism in both RA ${ }^{60}$ and SLE ${ }^{61}$. Beyond this, to our knowledge, very little is known about the clinical relevance of which effector mechanism is important for improving clinical response to rituximab. Further, disease-associated factors need careful consideration when evaluating the efficiency and resistance of anti-CD20 monoclonal antibodies, as discussed recently ${ }^{51}$.

\subsection{Blocking B-cell activating factor}

BAFF (also known as B-cell activating factor, BLyS) is a cytokine of TNF family, which binds to three receptors on B lymphocyte's surface: BLyS receptor 3 (BR3), transmembrane activator-1 and calcium modulator and cyclophillin ligand-interactor (TACl), and B-cell maturation antigen (BCMA). The suppression of the binding of BLyS to BR3 causes cell apoptosis and inhibition of the maturation of B-cells ${ }^{62}$. Therefore BAFF is considered an important target to manipulate $B$ cell function and/or survival.

The safety and efficacy of Belimumab, a monoclonal antibody that antagonises BAFF, was evaluated in two clinical trials involving more than 800 patients in each trial: BLISS $52{ }^{63}$ and BLISS $76{ }^{64}$. Both trials met their primary endpoints and at least some secondary endpoints ${ }^{24}$. An important caveat is that the most common features of patients in these large Phase III trials were skin and joint manifestations and patients with prior exposure to B-cell depletion were excluded. The primary endpoint of the studies was a composite score, the SLE 
Responder Index, which comprises a fall in Systemic Lupus Erythematosus Disease Activity Index of 4 points, no new BILAG A or B scores, and no change in the physician's global assessment. It met the primary endpoint with a $10 \%$ and $14 \%$ absolute response difference over placebo. Interestingly, post-hoc analysis have shown significant reduction in naive and activated B-cells, as well as plasma cells, in belimumab-treated patients ${ }^{65}$. These patients also experienced significant reductions in IgG and autoantibodies and improvement in C3 and C4 levels, whereas preexisting antipneumococcal or anti-tetanus toxoid antibody levels were not substantially affected ${ }^{65}$.

Atacicept is a fusion protein that antagonises the effects of BAFF and another cytokine known as APRIL. Both BAFF and APRIL levels are increased in SLE patients, suggesting that dual blockade by atacicept may be more potent than blockading BAFF alone; it has also the benefit of targeting long-lived plasma cells. A randomised Phase II/III trial of atacicept in non-renal SLE failed to achieve primary endpoint (reducing the number of SLE patients who had a new flare) in the $75 \mathrm{mg}$ arm compared with placebo. However, in a post-hoc analysis patients in the higher dose arm $(150 \mathrm{mg})$ who completed the study showed significantly fewer flares and time to first flare ${ }^{66}$. A significant reduction in antidsDNA antibodies, plasma cells and the mean IgG concentration in the atacicept groups was observed in this study. A separate study of renal patients was stopped because three patients given atacicept developed significant infections 67. However, more detailed analysis of the data showed these infections were linked to significant hypogammaglobulinemia induced by the mycophenolate given two weeks before any atacicept was administered.

Phase II trials of Blisibimod, an anti-BAFF monoclonal antibody, have encouraging results and there is an ongoing Phase III trial with a deliberate focus on patients with more active disease ${ }^{64}$.

Tabalumab is a fully human IgG4 monoclonal antibody that binds and neutralises both membrane and soluble BAFF. Two phase III studies, ILLUMINATE-1 68 and ILLUMINATE-2 ${ }^{68}$, evaluated the efficacy and safety of subcutaneous doses of tabalumab plus standard-of-care versus placebo plus standard-of-care in patients with active SLE. Patients received a loading dose 
of $240 \mathrm{mg}$ at week 0 and followed by $120 \mathrm{mg}$ every 2 weeks, $120 \mathrm{mg}$ every 4 weeks or placebo. ILLUMINATE 1 did not meet efficacy endpoints. However, the primary outcome (achieving SLE Responder Index 5 improvement at week 52) was met with in the group receiving 120 mg every 2 weeks in ILLUMINATE 2 , although it failed to met other key secondary endpoints ${ }^{68}$. An important point to consider though is the design of ILLUMINATE-1 stipulated that new, increased or decreased standard of care medications would define a patient as non-responder, whereas in ILLUMINATE 2 only new or increased medications determined non-response ${ }^{68}$. In both studies, significant reductions in total Bcells, immunoglobulins and anti-dsDNA antibodies levels and increases in C3 and C4 were observed with tabalumab.

\section{4) Combining anti-CD20 and anti-B-cell activating factor antibodies}

Cambridge and colleagues have shown that rituximab-based BCD leads to significant increases in serum BAFF levels in both RA 69 and SLE 70 . An important observation though is that the low B-cell counts was associated with the high serum BAFF levels ${ }^{70}$, due to simply relative lack of BAFF-receptor expressing B cells. During an 18-month follow-up period, a low BAFF state after B-cell depletion appeared to be associated with a favourable clinical outcome. High BAFF levels after B-cell depletion and repopulation of peripheral $B$ cells distinguished those patients with relapsing disease from those remaining in remission. Further, serum BAFF levels during relapse after B-cell depletion with Rituximab were significantly elevated in comparison with disease flare prior to treatment and correlate positively with levels of anti-dsDNA antibodies (prior and post treatment) ${ }^{70}$. Therefore, BAFF may have a role in disease flare after B-cell depletion, hence, special attention should be given to sequential B-cell depletion, where increasing BAFF levels and rising dsDNA antibody levels may promote a disease flare even at low B-cell numbers ${ }^{70}$.

BEAT-LUPUS, a randomised clinical trial is being undertaken at University College London Hospital and other centres, it will evaluate the potential of belimumab to prevent flares in patients with SLE after rituximab-based B-cell depletion therapy. 


\subsection{Other targets to achieve B-Cell depletion}

Epratuzumab is a monoclonal antibody that targets CD22 antigen on B cells. CD22 is considered to influence B-cell activation and migration. Dorner et al. published the first open label study in 2006 in which 14 patients with moderately active SLE were treated with four doses of i.v. epratuzumab $(360 \mathrm{mg} / \mathrm{m} 2)$ with antihistamine (but no steroids) as premedication, fortnightly. Total BILAG disease scores improved by $\geq 50 \%$ at $6,10,18$ and 32 weeks post treatment (77, 71 and $38 \%$, respectively). At the final 32 -week evaluation, statistically significant improvement in total BILAG score was observed, with $15 \%$ of the patients achieving $\geq 50 \%$ improvement ${ }^{71}$. The results of two Phase II RCTs where Epratuzumab significantly improved disease activity in patients with moderate-to-severe SLE ${ }^{72}, 73$. These studies were successfully viewed as a proof of concept in the efficacy of Epratuzumab in treating SLE and have led to further Phase III studies. However, first data from two phase 3 RCT multicenter studies of epratuzumab in active, moderate-to-severe SLE failed to meet its endpoints [(EMBODY $1^{\mathrm{TM}}$ (SL0009, NCT01262365) and EMBODY $2^{\mathrm{TM}}$ (SL0010, NCT01261793)] ${ }^{74}$.

Anti-CD19 targeting is potentially a new possible treatment, justified by its role in the threshold for BCR activation and by directly targeting autoantibodysecreting plasmablasts, plasma cells and early B-cells not targeted by antiCD20 therapy. Anti-CD19 targeting is being discussed as a new possible treatment, justified by its role in the threshold for BCR activation and by directly targeting autoantibody-secreting plasmablasts, plasma cells and early B-cells not targeted by anti-CD20 therapy. Successful targeting of CD19+ plasma cells in patients with SLE may target diverse range B-cell subpopulations compared to anti-CD20 mAbs. Immunological consequences of anti-CD19 therapy in vivo would also therefore warrant careful evaluation ${ }^{75}$.

\section{Expert commentary:}


B-cell depletion was clearly a sensible aproach for patients with RA and SLE since B-cells have major roles in their aetiopathogenesis. Rituximab, introduced in 1997 for the treatment of non-Hodgkin's lymphoma has established itself as a widely-used and effective treatment in rheumatoid factor positive and/or antiCCP positive RA patients. (It is however less successful in seronegative RA). Its success has been based on successful clinical trials following the original observations of Edwards and Cambridge. In contrast Rituximab, although considered to be useful by phyisicians who have used it, has not been as widely utilised in SLE because two major clinical trials did not meet primary end points. However, it is evident that trial design played a major part in this apparent lack of response. Several post hoc analyses have shown "hints and allegations" about its potential utility. Rituximab is now being used in a clinical trial in combination with Belimumab[Office1] which, having been approved by the FDA in 2012 was approved very recently by NICE. Belimumab blocks the B-cell activating factor BAFF. There are data to suggest that in some lupus patients following Rituximab there is a rise in BAFF which provides the academic rationale to treat patients with Rituximab followed by Belimumab.

Fully humanised B-cell depleting agents eg Ocrelizumab and Ofatumumab have been tried in clinical trials of patients with RA and SLE but to date are not widely used. A clinical trial of Ocrelizumab was stopped because of concerns about infections although these seemed to have been largely confined to patients living in the Far East.

\section{Five-year view:}

The use of B-cell depletion in rheumatoid arthritis is secure and the key questions here relate to whether newer forms of anti-CD20 mAbs will be even more effective with even fewer side-effects. Determining whether or not B-cell depletion has a part to play in the treatment of lupus is critically dependent upon the success of further clinical trials and this in turn depends upon improving clinical trial design and in ensuring the training of testing physicians wishing to participate in lupus clinical trials. 
The newer anti-CD20 mAbs are focused on improving the efficicency of B-cell depletion. These so-called type II anti-CD20 mAbs (eg Obinutuzamab) do not appear to lead to the clustering of CD20 molecules on the B-cell surface and are therefore less rapidly internalised compared to Rituximab (a type I antiCD20 monoclonal). Studies using this drug have been shown to be successful in chronic lymphocytic leukaemia and it is hoped that trials just started in lupus will also be successful.

In addition to giving anti-CD20 mAbs on their own, there is increasing interest in the notion that combinations of monoclonals will prove to be even more successful and there are high hopes for the Rituximab and Benlysta trials going on in the UK and United States. However this is one crystal ball which remains very hard to read!

7. Key Issues

- B cells are fundamental in the pathogenesis of rheumatoid arthritis (RA) and systemic lupus erythematosus (SLE).

- B-cell depletion therapy (BCDT) has been successfully used to treat RA.

- Although used to treat patients with refractory SLE in clinical practice, rituximab failed to proove its efficacy on two randomized controlled trials, probably owing to poor trial design.

- Newer anti-CD20 monoclonals may prove more eficient than rituximab at inducing B-cell depletion.

- Elevated B-cell activating factor levels after BCDT are associated with high anti-dsDNA antibodies, which provides the rationale for sequential therapy with Belimumab after treatment with rituximab. 


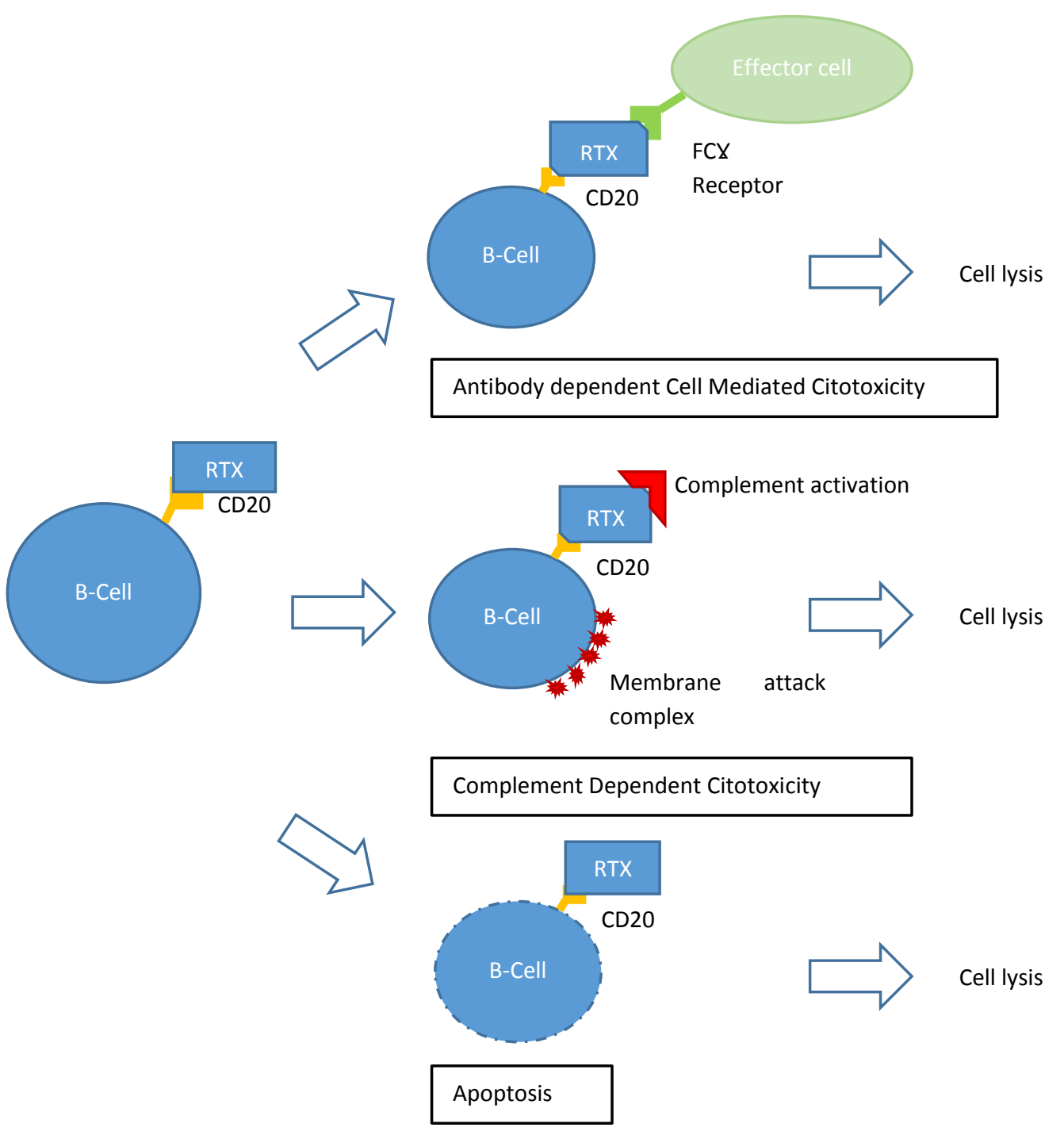


Figure 1 - Schematic illustrating rituximab mechanisms of action. Rituximab binds to $C D 20+B$ cells triggering effector mechanisms: antibody-dependent cell cytotoxicity, complement-dependent cellular cytotoxicity and direct cell death. B cells opsonised with rituximab are recognised as 'foreign' attracting complement deposition and/or engagement with FcyR-bearing effector cells that induce cellular cytotoxicity. Not shown in the fiugure is another important effector mechanism antibody-dependent cell phagocytosis.

\section{Reference:}

1. Edwards JC and Cambridge G. B-cell targeting in rheumatoid arthritis and other autoimmune diseases. Nat Rev Immunol. 2006; 6: 394-403.

2. Lisnevskaia L, Murphy G and Isenberg D. Systemic lupus erythematosus. Lancet. 2014; 384: 1878-88.

3. Dorner T, Kinnman N and Tak PP. Targeting B cells in immune-mediated inflammatory disease: a comprehensive review of mechanisms of action and identification of biomarkers. Pharmacol Ther. 2010; 125: 464-75.

4. Rahman A and Isenberg DA. Systemic lupus erythematosus. N Engl J Med. 2008; 358: 929-39.

5. Dorner T, Giesecke C and Lipsky PE. Mechanisms of B cell autoimmunity in SLE. Arthritis Res Ther. 2011; 13: 243.

6. Isaacs JD. Therapeutic T-cell manipulation in rheumatoid arthritis: past, present and future. Rheumatology (Oxford). 2008; 47: 1461-8.

7. Sidiropoulos PI and Boumpas DT. Lessons learned from anti-CD40L treatment in systemic lupus erythematosus patients. Lupus. 2004; 13: 391-7.

8. Edwards JC, Cambridge $\mathrm{G}$ and Abrahams VM. Do self-perpetuating B lymphocytes drive human autoimmune disease? Immunology. 1999; 97: 188-96.

9. Batten M, Groom J, Cachero TG, et al. BAFF mediates survival of peripheral immature B lymphocytes. J Exp Med. 2000; 192: 1453-66.

10. Cambridge G, Leandro MJ, Edwards JC, et al. Serologic changes following B lymphocyte depletion therapy for rheumatoid arthritis. Arthritis Rheum. 2003; 48: 2146-54.

11. Mclnnes IB and Schett G. The pathogenesis of rheumatoid arthritis. N Engl J Med. 2011; 365: 2205-19.

12. Dias S and Isenberg D. Advances in systemic lupus erythematosus. Medicine (Baltimore). 2014; 42: 126-33.

13. McLaughlin P, Grillo-Lopez AJ, Link BK, et al. Rituximab chimeric anti-CD20 monoclonal antibody therapy for relapsed indolent lymphoma: half of patients respond to a four-dose treatment program. $J$ Clin Oncol. 1998; 16: 2825-33.

14. Edwards JC and Cambridge G. Sustained improvement in rheumatoid arthritis following a protocol designed to deplete B lymphocytes. Rheumatology (Oxford). 2001; 40: 205-11.

15. Edwards JC, Szczepanski L, Szechinski J, et al. Efficacy of B-cell-targeted therapy with rituximab in patients with rheumatoid arthritis. N Engl J Med. 2004; 350: 2572-81.

16. Leandro MJ, Edwards JC, Cambridge G, Ehrenstein MR and Isenberg DA. An open study of B lymphocyte depletion in systemic lupus erythematosus. Arthritis Rheum. 2002; 46: 2673-7.

17. Looney RJ, Anolik JH, Campbell D, et al. B cell depletion as a novel treatment for systemic lupus erythematosus: a phase I/II dose-escalation trial of rituximab. Arthritis Rheum. 2004; 50: 2580-9.

18. Diaz-Lagares C, Croca S, Sangle S, et al. Efficacy of rituximab in 164 patients with biopsy-proven lupus nephritis: pooled data from European cohorts. Autoimmun Rev. 2012; 11: 357-64. 
19. Lu TY, Ng KP, Cambridge G, et al. A retrospective seven-year analysis of the use of B cell depletion therapy in systemic lupus erythematosus at University College London Hospital: the first fifty patients. Arthritis Rheum. 2009; 61: 482-7.

20. Merrill JT, Neuwelt CM, Wallace DJ, et al. Efficacy and safety of rituximab in moderately-toseverely active systemic lupus erythematosus: the randomized, double-blind, phase II/III systemic lupus erythematosus evaluation of rituximab trial. Arthritis Rheum. 2010; 62: 222-33.

21. Rovin BH, Furie R, Latinis $\mathrm{K}$, et al. Efficacy and safety of rituximab in patients with active proliferative lupus nephritis: the Lupus Nephritis Assessment with Rituximab study. Arthritis Rheum. 2012; 64: 1215-26.

22. Favas C and Isenberg DA. B-cell-depletion therapy in SLE--what are the current prospects for its acceptance? Nat Rev Rheumatol. 2009; 5: 711-6.

23. Reddy V, Jayne D, Close D and Isenberg D. B-cell depletion in SLE: clinical and trial experience with rituximab and ocrelizumab and implications for study design. Arthritis Res Ther. 2013; 15 Suppl 1: S2.

24. Isenberg DA and Merrill JT. Why, why, why de-lupus (does so badly in clinical trials). Expert Rev Clin Immunol. 2016; 12: 95-8.

25. Terrier B, Amoura Z, Ravaud P, et al. Safety and efficacy of rituximab in systemic lupus erythematosus: results from 136 patients from the French Autolmmunity and Rituximab registry. Arthritis Rheum. 2010; 62: 2458-66.

26. Albert D, Dunham J, Khan S, et al. Variability in the biological response to anti-CD20 B cell depletion in systemic lupus erythaematosus. Ann Rheum Dis. 2008; 67: 1724-31.

27. Furtado J and Isenberg DA. Reprint of: B cell elimination in systemic lupus erythematosus. Clin. Immunol. 146(2) 90-103. Clin Immunol. 2013; 148: 344-58.

28. Reddy V, Martinez L, Isenberg DA, Leandro MJ and Cambridge G. Pragmatic treatment of patients with Systemic Lupus Erythematosus with rituximab: Long-term effects on serum immunoglobulins. Arthritis Care Res (Hoboken). 2016.

29. Clifford DB, Ances B, Costello C, et al. Rituximab-associated progressive multifocal leukoencephalopathy in rheumatoid arthritis. Arch Neurol. 2011; 68: 1156-64.

30. Leandro MJ, Edwards JC and Cambridge G. Clinical outcome in 22 patients with rheumatoid arthritis treated with B lymphocyte depletion. Ann Rheum Dis. 2002; 61: 883-8.

31. Edwards JC, Leandro MJ and Cambridge G. B lymphocyte depletion therapy with rituximab in rheumatoid arthritis. Rheum Dis Clin North Am. 2004; 30: 393-403, viii.

32. Leandro MJ, Cambridge G, Ehrenstein MR and Edwards JC. Reconstitution of peripheral blood B cells after depletion with rituximab in patients with rheumatoid arthritis. Arthritis Rheum. 2006; 54: 61320.

33. Ramos-Casals M, Soto MJ, Cuadrado MJ and Khamashta MA. Rituximab in systemic lupus erythematosus: A systematic review of off-label use in 188 cases. Lupus. 2009; 18: 767-76.

34. Gregersen JW and Jayne DR. B-cell depletion in the treatment of lupus nephritis. Nat Rev Nephrol. 2012; 8: 505-14.

35. Reddy VaL, M. Variability in clinical and biological response to rituximab in autoimmune diseases: an opportunity for personalized therapy? International Journal of Clinical Rheumatology. 2014; 9: 279.

36. Cambridge G, Leandro MJ, Teodorescu M, et al. B cell depletion therapy in systemic lupus erythematosus: effect on autoantibody and antimicrobial antibody profiles. Arthritis Rheum. 2006; 54: 3612-22.

37. Vital EM, Dass S, Buch $\mathrm{MH}$, et al. B cell biomarkers of rituximab responses in systemic lupus erythematosus. Arthritis Rheum. 2011; 63: 3038-47.

38. Kattah AG and Fervenza FC. Rituximab: emerging treatment strategies of immune-mediated glomerular disease. Expert Rev Clin Immunol. 2012; 8: 413-21.

39. Moroni G, Raffiotta F, Trezzi B, et al. Rituximab vs mycophenolate and vs cyclophosphamide pulses for induction therapy of active lupus nephritis: a clinical observational study. Rheumatology (Oxford). 2014; 53: 1570-7.

40. Pepper R, Griffith M, Kirwan C, et al. Rituximab is an effective treatment for lupus nephritis and allows a reduction in maintenance steroids. Nephrol Dial Transplant. 2009; 24: 3717-23.

41. Ezeonyeji AN and Isenberg DA. Early treatment with rituximab in newly diagnosed systemic lupus erythematosus patients: a steroid-sparing regimen. Rheumatology (Oxford). 2012; 51: 476-81. 
42. Condon MB, Ashby D, Pepper RJ, et al. Prospective observational single-centre cohort study to evaluate the effectiveness of treating lupus nephritis with rituximab and mycophenolate mofetil but no oral steroids. Ann Rheum Dis. 2013; 72: 1280-6.

43. Flores-Borja F, Bosma A, Ng D, et al. CD19+CD24hiCD38hi B cells maintain regulatory $T$ cells while limiting TH1 and TH17 differentiation. Sci Transl Med. 2013; 5: 173 ra23.

44. Menon M, Blair PA, Isenberg DA and Mauri C. A Regulatory Feedback between Plasmacytoid Dendritic Cells and Regulatory B Cells Is Aberrant in Systemic Lupus Erythematosus. Immunity. 2016; 44: 683-97.

45. Dias SS, Rodriguez-Garcia V, Nguyen H, Pericleous C and Isenberg D. Longer duration of B cell depletion is associated with better outcome. Rheumatology (Oxford). 2015; 54: 1876-81.

46. Vital EM, Rawstron AC, Dass S, et al. Reduced-dose rituximab in rheumatoid arthritis: efficacy depends on degree of B cell depletion. Arthritis Rheum. 2011; 63: 603-8.

47. Vital EM, Dass S, Buch MH, Rawstron AC and Emery P. An extra dose of rituximab improves clinical response in rheumatoid arthritis patients with initial incomplete B cell depletion: a randomised controlled trial. Ann Rheum Dis. 2015; 74: 1195-201.

48. Breedveld F, Agarwal S, Yin M, et al. Rituximab pharmacokinetics in patients with rheumatoid arthritis: B-cell levels do not correlate with clinical response. J Clin Pharmacol. 2007; 47: 1119-28.

49. Reddy V, Croca S, Gerona D, et al. Serum rituximab levels and efficiency of B cell depletion: differences between patients with rheumatoid arthritis and systemic lupus erythematosus. Rheumatology (Oxford). 2013; 52: 951-2.

50. Glennie MJ, French RR, Cragg MS and Taylor RP. Mechanisms of killing by anti-CD20 monoclonal antibodies. Mol Immunol. 2007; 44: 3823-37.

51. Reddy V, Dahal LN, Cragg MS and Leandro M. Optimising B-cell depletion in autoimmune disease: is obinutuzumab the answer? Drug Discov Today. 2016; 21: 1330-8.

52. Lim SH, Beers SA, French RR, Johnson PW, Glennie MJ and Cragg MS. Anti-CD20 monoclonal antibodies: historical and future perspectives. Haematologica. 2010; 95: 135-43.

53. Taylor PC, Quattrocchi E, Mallett S, Kurrasch R, Petersen J and Chang DJ. Ofatumumab, a fully human anti-CD20 monoclonal antibody, in biological-naive, rheumatoid arthritis patients with an inadequate response to methotrexate: a randomised, double-blind, placebo-controlled clinical trial. Ann Rheum Dis. 2011; 70: 2119-25.

54. Thornton CC, Ambrose $\mathrm{N}$ and loannou Y. Ofatumumab: a novel treatment for severe systemic lupus erythematosus. Rheumatology (Oxford). 2015; 54: 559-60.

55. Bowles JA, Wang SY, Link BK, et al. Anti-CD20 monoclonal antibody with enhanced affinity for CD16 activates NK cells at lower concentrations and more effectively than rituximab. Blood. 2006; 108: 2648-54.

56. Beers SA, Chan $\mathrm{CH}$, James $\mathrm{S}$, et al. Type II (tositumomab) anti-CD20 monoclonal antibody out performs type I (rituximab-like) reagents in B-cell depletion regardless of complement activation. Blood. 2008; 112: 4170-7.

57. Tipton TR, Roghanian A, Oldham RJ, et al. Antigenic modulation limits the effector cell mechanisms employed by type I anti-CD20 monoclonal antibodies. Blood. 2015; 125: 1901-9.

58. Goede V, Klein C and Stilgenbauer S. Obinutuzumab (GA101) for the treatment of chronic lymphocytic leukemia and other B-cell non-hodgkin's lymphomas: a glycoengineered type II CD20 antibody. Oncol Res Treat. 2015; 38: 185-92.

59. Reddy V, Cambridge G, Isenberg DA, Glennie MJ, Cragg MS and Leandro M. Internalization of Rituximab and the Efficiency of B Cell Depletion in Rheumatoid Arthritis and Systemic Lupus Erythematosus. Arthritis \& rheumatology. 2015; 67: 2046-55.

60. Kastbom A, Coster L, Arlestig L, et al. Influence of FCGR3A genotype on the therapeutic response to rituximab in rheumatoid arthritis: an observational cohort study. BMJ Open. 2012; 2.

61. Anolik JH, Campbell D, Felgar RE, et al. The relationship of FcgammaRIlla genotype to degree of B cell depletion by rituximab in the treatment of systemic lupus erythematosus. Arthritis Rheum. 2003; 48: 455-9.

62. Cancro MP, D'Cruz DP and Khamashta MA. The role of B lymphocyte stimulator (BLyS) in systemic lupus erythematosus. J Clin Invest. 2009; 119: 1066-73.

63. Navarra SV, Guzman RM, Gallacher AE, et al. Efficacy and safety of belimumab in patients with active systemic lupus erythematosus: a randomised, placebo-controlled, phase 3 trial. Lancet. 2011; 377: 721-31. 
64. Furie R, Petri M, Zamani O, et al. A phase III, randomized, placebo-controlled study of belimumab, a monoclonal antibody that inhibits B lymphocyte stimulator, in patients with systemic lupus erythematosus. Arthritis Rheum. 2011; 63: 3918-30.

65. Stohl W, Hiepe F, Latinis KM, et al. Belimumab reduces autoantibodies, normalizes low complement levels, and reduces select B cell populations in patients with systemic lupus erythematosus. Arthritis Rheum. 2012; 64: 2328-37.

66. Isenberg D, Gordon C, Licu D, Copt S, Rossi CP and Wofsy D. Efficacy and safety of atacicept for prevention of flares in patients with moderate-to-severe systemic lupus erythematosus (SLE): 52-week data (APRIL-SLE randomised trial). Ann Rheum Dis. 2015; 74: 2006-15.

67. Ginzler EM, Wax S, Rajeswaran A, et al. Atacicept in combination with MMF and corticosteroids in lupus nephritis: results of a prematurely terminated trial. Arthritis Res Ther. 2012; 14: R33.

68. Isenberg DA, Petri M, Kalunian K, et al. Efficacy and safety of subcutaneous tabalumab in patients with systemic lupus erythematosus: results from ILLUMINATE-1, a 52-week, phase III, multicentre, randomised, double-blind, placebo-controlled study. Ann Rheum Dis. 2016; 75: 323-31.

69. Cambridge G, StohI W, Leandro MJ, Migone TS, Hilbert DM and Edwards JC. Circulating levels of B lymphocyte stimulator in patients with rheumatoid arthritis following rituximab treatment: relationships with B cell depletion, circulating antibodies, and clinical relapse. Arthritis Rheum. 2006; 54: 723-32.

70. Carter LM, Isenberg DA and Ehrenstein MR. Elevated serum BAFF levels are associated with rising anti-double-stranded DNA antibody levels and disease flare following B cell depletion therapy in systemic lupus erythematosus. Arthritis Rheum. 2013; 65: 2672-9.

71. Dorner T, Kaufmann J, Wegener WA, Teoh N, Goldenberg DM and Burmester GR. Initial clinical trial of epratuzumab (humanized anti-CD22 antibody) for immunotherapy of systemic lupus erythematosus. Arthritis Res Ther. 2006; 8: R74.

72. Wallace DJ, Kalunian K, Petri MA, et al. Efficacy and safety of epratuzumab in patients with moderate/severe active systemic lupus erythematosus: results from EMBLEM, a phase IIb, randomised, double-blind, placebo-controlled, multicentre study. Ann Rheum Dis. 2014; 73: 183-90.

73. Wallace DJ, Gordon C, Strand V, et al. Efficacy and safety of epratuzumab in patients with moderate/severe flaring systemic lupus erythematosus: results from two randomized, double-blind, placebo-controlled, multicentre studies (ALLEVIATE) and follow-up. Rheumatology (Oxford). 2013; 52: 1313-22.

74. Clowse ME, Wallace DJ, Furie RA, et al. Efficacy and Safety of Epratuzumab in Moderately to Severely Active Systemic Lupus Erythematosus: Results from the Phase 3, Randomized, Double-blind, Placebo-controlled Trials, EMBODY 1 and EMBODY 2. Arthritis \& rheumatology. 2016.

75. Mei HE, Schmidt S and Dorner T. Rationale of anti-CD19 immunotherapy: an option to target autoreactive plasma cells in autoimmunity. Arthritis Res Ther. 2012; 14 Suppl 5: S1. 